\title{
GLOBAL STRUCTURE IN VON NEUMANN ALGEBRAS
}

\author{
BY \\ EDWARD G. EFFROS(1)
}

1. Introduction. A von Neumann algebra on a separable Hilbert space has an essentially unique representation as a direct integral of factors. In this paper we shall investigate the extent to which such decompositions reduce the classification problem for von Neumann algebras to that for factors. G. W. Mackey [18] has studied the corresponding questions that arise in the direct integral theory for representations of a separable locally compact group $G$. The essential concept that he introduced was the dual $\hat{G}$ of $G$, the set of unitary equivalence classes of separable, irreducible, unitary representations of $G$. $\hat{G}$, together with a natural $\sigma$-algebra of sets (a "Borel structure"), may be used as an index space for direct integrals of irreducible representations. We follow Mackey's program by introducing canonical index spaces $\hat{\mathscr{F}}$ and $\tilde{\mathscr{F}}$ for direct integrals of factors.

Let $\mathfrak{S}_{n}$ be a fixed Hilbert space of dimension $n, 1 \leqq n \leqq \infty=\aleph_{0}$ and $\mathscr{A}_{n}$ be the set of all von Neumann algebras on $\mathfrak{H}_{n}$. In [5] we introduced a standard Borel structure on $\mathscr{A}_{n}$. Let $\mathscr{A}=\bigcup_{n=1}^{\infty} \mathscr{A}_{n}$ have the Borel structure generated by the structures on the $\mathscr{A}_{n}$. The relative structure on the set of factors $\mathscr{F}$ is standard [5, Corollary 3 of Theorem 3]. We define $\hat{\mathscr{F}}$ and $\tilde{\mathscr{F}}$ to be the spatial and algebraic isomorphism classes in $\mathscr{F}$, together with the quotient structures.

We have been unable to determine if $\hat{\mathscr{F}}$ and $\tilde{\mathscr{F}}$ are smooth, i.e., if they have countably separated structures. The problem is of considerable interest, as when a group $G$ has a nonsmooth dual, direct integrals provide one with representations of $G$ having unusual (i.e., nontype I) global structure (see [4, $\$ 4]$ ). In $\$ 2$ and $\S 3$ we show that points in $\hat{\mathscr{F}}$ and $\tilde{\mathscr{F}}$ are Borel, and that $\hat{\mathscr{F}}$ is smooth if and only if $\tilde{\mathscr{F}}$ is smooth. It follows that if there should be only countably many points in $\tilde{\mathscr{F}}$, $\tilde{\mathscr{F}}$ and $\hat{\mathscr{F}}$ would be smooth. At present only nine algebraically distinct nontype I factors have been identified (see [22], [23]).

The decomposition of a von Neumann algebra into factors induces a measure on $\hat{\mathscr{F}}$. We say that the algebra is centrally smooth if the complement of a null Borel set is countably separated. In $\S 5$, we show that such algebras are those of the form

$$
\sum_{m=0}^{\infty} \int \mathfrak{B}(\xi) d \mu_{m}(\xi) \otimes \mathfrak{P}_{m}
$$

Received by the editors May 24, 1965.

(1) Research supported partially by NR 043-325. 
where the $\mu_{m}$ are countably separated measures on $\hat{\mathscr{F}}$, almost every $\mathfrak{B}(\xi)$ is a factor in the equivalence class $\xi$, and the $\mathfrak{P}_{m}$ are various abelian von Neumann algebras. The proof uses a double integral technique of Mackey [16, Theorem 2.11]. In an attempt to make the latter more accessible, we have included a careful exposition of the theory in $\S 4$. Our approach gives some information even when the quotient measure is not countably separated (see Lemmas 4.4 and 4.5 ).

In $\S 6$ we consider the global pathology that might occur should $\hat{\mathscr{F}}$ be nonsmooth. Introducing the notion of global type, it becomes apparent that a von Neumann algebra of global type II could not be centrally smooth.

We are indebted to $R$. V. Kadison for several fruitful conversations on the material in $\S 6$, and to both him and J. Ringrose for ideas that resulted in Lemma 2.5. We have recently a manuscript from J. Feldman [6], in which he has proved the analogue of Theorem 2.8 for the representation and state spaces of separable $C^{*}$-algebras.

2. Subsets of $\mathscr{A}$. Let $\mathfrak{R}_{n}$ be the bounded linear operators on $\mathfrak{H}_{n}$. The weak, $\sigma$-weak, strong, and $\sigma$-strong topologies generate the same Borel structure on $\mathfrak{L}_{n}$, and the algebraic operations are Borel.

Let $G_{n}$ be the group of unitaries on $\mathfrak{H}_{n}$ with the strong topology. $G_{n}$ is a polonais topological group [3, Lemma 4], and defining $\phi_{n}: G_{n} \otimes \mathscr{A}_{n} \rightarrow \mathscr{A}_{n}$ by $\phi_{n}(U, \mathfrak{A})$ $=U \mathfrak{A} U^{-1}$, we obtain a transformation group.

\section{LeMMA 2.1. $\phi_{n}$ is Borel.}

Proof. Let $\mathfrak{A} \rightarrow A_{i}(\mathfrak{H})$ be Borel choice functions on $\mathscr{A}_{n}$ with $A_{i}(\mathfrak{A})$ weakly dense in $\mathfrak{A}_{1}$ for each $\mathfrak{A}$ [5, Corollary of Theorem 2]. If $f \in \mathfrak{R}_{n^{*}}$,

$$
\left\|f \mid \phi_{n}(U, \mathfrak{A})\right\|=\sup \left\{\left|f\left(U A_{i}(\mathfrak{A}) U^{*}\right)\right|: i=1,2, \cdots\right\} .
$$

As multiplication is Borel, $(U, \mathfrak{A}) \rightarrow f\left(U A_{i}(\mathfrak{A}) U^{*}\right)$ is Borel. Thus

$$
(U, \mathfrak{A}) \rightarrow\left\|f \mid \phi_{n}(U, \mathfrak{R})\right\|
$$

is Borel, and from [5, Theorem 1], $\phi_{n}$ is Borel.

Let $\mathfrak{I}_{n}$ be the scalar multiples of the identity operator on $\mathfrak{H}_{n} \cdot \mathfrak{A} \rightarrow \mathfrak{A} \otimes \mathfrak{I}_{\infty}$ may be realized as a map $\theta$ of $\mathscr{A}_{\text {into }} \mathscr{A}_{\infty}$ as follows. For each $n$, choose an infinite sequence of isometries $U_{i n}$ of $\mathfrak{S}_{n}$ into $\mathfrak{H}_{\infty}$, for which the projections $E_{i n}=U_{i n} U_{i n}^{*}$ are orthogonal and $\Sigma_{i} E_{i n}=I$. Define $\theta_{n}^{\prime}: \mathfrak{L}_{n} \rightarrow \mathfrak{L}_{\infty}$ by $\theta_{n}^{\prime}(A)=\Sigma_{i} U_{i n} A U_{i n}^{*}$. $\theta_{n}^{\prime}$ is a $\sigma$-weakly continuous isomorphism of $\mathfrak{L}_{n}$ into $\mathfrak{L}_{\infty}$ which preserves the identity. If $\mathfrak{A} \in \mathscr{A}_{n}$, let $\theta_{n}(\mathfrak{A})=\left\{\theta_{n}^{\prime}(A): A \in \mathfrak{A}\right\}$. As $\theta_{n}^{\prime}$ is $\sigma$-weakly continuous, it is the adjoint of a map $\theta_{n^{*}}^{\prime}: \mathfrak{L}_{\infty^{*}} \rightarrow \mathfrak{L}_{n^{*}}$. As $\theta_{n}^{\prime}$ is an isometry, we have for $f \in \mathfrak{L}_{i c}$,

$$
\left\|f\left|\theta_{n}(\mathfrak{Z})\|=\| \theta_{n^{*}}^{\prime} f\right| \mathfrak{A}\right\|,
$$

and from [5, Theorem 1], $\theta_{n}$ is Borel. We let $\theta=\bigcup_{n} \theta_{n}$. 
THEOREM 2.2. If $\mathfrak{A} \in \mathscr{A}$, the spatial and algebraic equivalence classes [ $\mathfrak{A}]$ and $[[\mathfrak{O}]]$ containing $\mathfrak{A}$ are Borel.

Proof. If $\mathfrak{A} \in \mathscr{A}_{n}$, let $G_{n}(\mathfrak{U})$ be the stabilizer subgroup of $\mathfrak{A}$ in $G_{n}$, i.e., those unitary $U$ with $U \mathfrak{A} U^{*}=\mathfrak{A}$. As $G_{n}(\mathfrak{A})$ consists of the $U \in G_{n}$ with $U \mathfrak{A} U^{*} \subseteq \mathfrak{A}$ and $U^{*} \mathfrak{A} U \subseteq \mathfrak{A}, G_{n}(\mathfrak{A})$ is closed in $G_{n}$. From [3, Lemma 3], there exists a Borel set $T$ in $G_{n}$ intersecting each left coset of $G_{n}(\mathfrak{U})$ in one point. We have

$$
[\mathfrak{U}]=\phi_{n}\left(G_{n} \times\{\mathfrak{U}\}\right)=\phi_{n}(T \times\{\mathfrak{U}\}) .
$$

As $\dot{\phi}_{n}$ is Borel and one-to-one on the standard Borel space $T \times\{\mathfrak{A}\}$, [थ्A] is Borel (see [18, Theorem 3.2]). von Neumann algebras with purely infinite commutants on a separable Hilbert space are algebraically isomorphic if and only if they are spatially isomorphic, hence

$$
[[\mathfrak{A}]]=\theta^{-1}([\theta(\mathfrak{A})])
$$

and $[[\mathfrak{U}]]$ is Borel.

Let $\hat{A}$ and $\tilde{A}$ be the spatial, respectively algebraic, equivalence classes in $\mathscr{A}$, with the quotient Borel structures.

Corollary 2.3. Points in $\hat{\mathscr{A}}$ and $\tilde{\mathscr{A}}$ are Borel.

Corollary 2.4. The set $\mathscr{A}_{I}$ of von Neumann algebras of type I is Borel.

Proof. Let $\mathscr{B}$ denote the properly infinite type I von Neumann algebras. To within algebraic equivalence, there are only countably many such algebras. Thus $\mathscr{B}$ is a countable union of algebraic equivalence classes, and is Borel. A von Neumann algebra $\mathfrak{A}$ is of type $I$ if and only if $\theta(\mathfrak{U})^{\prime}$ is properly infinite and type $I$. Thus

$$
\mathscr{A}_{I}=\theta^{-1}\left(\mathscr{B}^{\prime}\right) \text {, }
$$

and as ' is a Borel isomorphism of $\mathscr{A}$ into itself [5, Theorem 3], $\mathscr{A}_{I}$ is Borel.

Let $\mathscr{F}_{n}$ be the factors on $\mathfrak{S}_{n}$, and $\mathscr{F}=\bigcup_{n=1}^{\infty} \mathscr{F}_{n}$. From $[5$, Corollary 3 of Theorem 3] $\mathscr{F}_{n}$ is a Borel subset of $\mathscr{A}_{n}$, hence $\mathscr{F}$ is a Borel subset of $\mathscr{A}$. We wish to show that $\mathscr{F}_{\text {fin }}$, the finite factors, is also Borel.

If $\mathfrak{A}$ is a von Neumann algebra and $A \in \mathfrak{A}$, let $\kappa_{\mathfrak{A}}(A)$ be the weak closure of finite convex sums of elements of the form $U A U^{*}$, with $U$ a unitary in $\mathfrak{A}$.

LEMMA 2.5. Let $\mathfrak{A}$ be a factor and $E \neq 0, I$ be a projection in $\mathfrak{A}$. $\mathfrak{A}$ is infinite if and only if $0 \in \kappa_{\mathfrak{H}}(E)$ or $0 \in \kappa_{\mathfrak{H}}(I-E)$.

Proof. If $\mathfrak{A}$ is finite, and $\tau$ is the normalized trace on $\mathfrak{A}, \tau$ must be constant on each of the sets $\kappa_{\mathfrak{Q}}(E)$ and $\kappa_{\mathfrak{q}}(I-E)$. As $\tau(E)$ and $\tau(I-E)$ are nonzero, $0 \notin \kappa_{\mathfrak{A}}(E)$ and $0 \notin \kappa_{\mathfrak{A}}(I-E)$. 
If $\mathfrak{A}$ is infinite, say that $I-E$ is infinite. For any $n$, we may choose partial isometries $U_{1}, \cdots, U_{n}$ with $U_{i}^{*} U_{i}=E$ and $U_{i} U_{i}^{*}=E_{i}$ orthogonal projections with $E_{i} \leqq I-E$. Let

$$
V_{i}=U_{i}+U_{i}^{*}+\left[I-\left(E+E_{i}\right)\right] .
$$

Then $V_{i}$ is unitary, and

$$
\left\|\frac{1}{n} \sum_{i=1}^{n} V_{i} E V_{i}^{*}\right\|=\frac{1}{n}
$$

As $n$ is arbitrary, $0 \in \kappa_{\mathfrak{a}}(E)$. Similarly, if $E$ is infinite, $0 \in \kappa_{\mathfrak{A}}(I-E)$. As one or the other is infinite, we are done.

LEMMA 2.6. There exist Borel choice functions $\mathfrak{A} \rightarrow A_{i}(\mathfrak{A})$ and $\mathfrak{A} \rightarrow U_{i}(\mathfrak{A})$ on $\mathscr{A}$ with the $A_{i}(\mathfrak{U})$ strongly dense in $\mathfrak{U}_{S A, 1}$, the self-adjoint elements $A \in \mathfrak{U}$ with $\|A\| \leqq 1$, and the $U_{i}(\mathfrak{U})$ strongly dense in the unitaries of $\mathfrak{A}$, for all $\mathfrak{A} \in \mathscr{A}$.

Proof. From [5, Theorem 2], there exist Borel $\mathfrak{U} \rightarrow C_{j}(\mathfrak{U})$ with the $C_{j}(\mathfrak{U})$ weakly dense in $\mathfrak{U}_{1}$. As the adjoint operation is weakly continuous, $\mathfrak{U} \rightarrow B_{j}(\mathfrak{U})=\frac{1}{2}\left[C_{j}(\mathfrak{U})^{*}+C_{j}(\mathfrak{U})\right]$ is Borel, and the $B_{j}(\mathfrak{U})$ are weakly dense in $\mathfrak{U}_{S A}{ }_{1}$. Let $A_{i}(\mathfrak{U})$ be an enumeration of the finite sums of the form $\Sigma r_{j} B_{j}(\mathfrak{A})$, where the $r_{j}$ are positive rationals with $\Sigma_{j} r_{j}=1$. For each $\mathfrak{U}$, the strong closure of the $A_{i}(\mathfrak{U})$ is convex, hence it is weakly closed and coincides with $\mathfrak{A}_{S A, 1}$.

Define $f$ on the closed interval $[-1,1]$ by $f(t)=\exp (i \pi t)$, and $g$ on the unit circle by letting $g(z)$ be the unique real $t$ with $-1 \leqq t<1$ and $\exp i \pi t=z$. If $A \in \mathfrak{A}_{S A, 1}, f(A)$ is unitary, and if $U$ is unitary, $g(U) \in \mathfrak{A}_{S A, 1}$ and $f(g(U))=(f \circ g)(U)=U$. Thus $g$ maps $\mathfrak{U}_{S_{A}, 1}$ onto the unitaries of $\mathfrak{A}$. As $A \rightarrow f(A)$ is strongly continuous $\left[12\right.$, p. 232], the operators $U_{i}(\mathfrak{U})=f\left(A_{i}(\mathfrak{U})\right)$ are strongly dense in the unitaries. From the argument used in [5, Theorem 5], $\mathfrak{U} \rightarrow U_{i}(\mathfrak{U})$ is Borel.

LEMMA 2.7. There exists a Borel choice function $\mathfrak{U} \rightarrow E(\mathfrak{U})$ on $\mathscr{A}$ with $E(\mathfrak{U})$ a projection, $E(\mathfrak{U}) \neq 0$, I for $\mathfrak{A} \neq \mathfrak{I}_{n}, n=1,2, \cdots \infty$.

Proof. If $A$ is a self-adjoint operator on $\mathfrak{S}_{n}$, let

$$
\begin{aligned}
& m(A)=\inf \{A x \cdot x:\|x\|=1\}, \\
& M(A)=\sup \{A x \cdot x:\|x\|=1\}, \\
& \omega(A)=M(A)-m(A) .
\end{aligned}
$$

Letting $x_{i}$ be dense in the unit ball of $\mathfrak{H}_{n}$,

$$
m(A)=\inf \left\{A x_{i} \cdot x_{i}: i=1,2, \cdots\right\},
$$

hence $m$, and similarly $M$, are Borel on $\mathfrak{L}_{n, s_{A}} \cdot \omega(A)=0$ if and only if $A$ is a 
scalar multiple of $I$. Let $\mathfrak{A} \rightarrow A_{i}(\mathfrak{H})$ be Borel choice functions on $\mathfrak{A}$ with $A_{i}(\mathfrak{H})$ weakly dense in $\mathfrak{A}_{S A, 1}$. If $\omega\left(A_{i}(\mathfrak{A})\right)=0$ for all $i, \mathfrak{A}=\mathfrak{I}_{n}$ for some $n$. Define a Borel choice function $B$ by $B\left(\mathfrak{I}_{n}\right)=0$ for all $n$, and if $\mathfrak{A} \neq \mathfrak{I}_{n}, B(\mathfrak{H})=A_{i}(\mathfrak{H})$, where $i$ is the first integer with $\omega\left(A_{i}(\mathfrak{H})\right) \neq 0$. Let $E(\mathfrak{H})=f^{\mathfrak{I}}(B(\mathfrak{A}))$, where $f^{\mathfrak{A}}$ is the characteristic function of the closed interval

$$
\left[\frac{1}{2} m(B(\mathfrak{A}))+\frac{1}{2} M(B(\mathfrak{H})), 1\right] .
$$

Then $E(\mathfrak{H}) \neq 0, I$ for $\mathfrak{A} \neq \mathfrak{J}_{n}$, and it suffices to show that $\mathfrak{A} \rightarrow f^{\mathfrak{A}}(B(\mathfrak{A}))$ is Borel.

Let $\mathfrak{C}$ be the continuous real-valued functions on $[-2,1]$ with the uniform norm. The map

$$
\mathfrak{C} \times \mathfrak{L}_{n, S A, 1} \rightarrow \mathfrak{L}_{n}:(f, A) \rightarrow f(A)
$$

is continuous in the first variable, and Borel in the second (see the proof of [5, Theorem 5]), hence it is jointly Borel (see [15, Lemma 9.2], [13,§27V]). Given $a \in[-1,1]$ and a positive integer $k$, define $f_{k}^{a} \in \mathbb{C}$ by $f_{k}^{a}(t)=0$ for $t \leqq a-1 / k$, $f_{k}^{a}(t)=1$ for $t \geqq a$, and letting $f_{k}^{a}(t)$ be linear on intermediate points. $a \rightarrow f_{k}^{a}$ is a continuous map of $[-1,1]$ into $\mathfrak{C}$, hence letting

$$
\mu(\mathfrak{A})=\frac{1}{2} m(B(\mathfrak{U}))+\frac{1}{2} M(B(\mathfrak{H})),
$$

$\mathfrak{A} \rightarrow f_{k}^{\mu(\mathfrak{l})}(B(\mathfrak{A}))$ is Borel for each $k$. Fixing $\mathfrak{A}$, the $f_{k}^{\mu(\mathfrak{I})}$ are uniformly bounded and converge point-wise to $f^{\mathfrak{A}}$, hence $f_{k}^{\mu(\mathfrak{l} \mathfrak{f}}(B(\mathfrak{A})) \rightarrow f^{\mathfrak{I}}(B(\mathfrak{A}))$ weakly. Thus $\mathfrak{A} \rightarrow f^{\mathfrak{A}}(B(\mathfrak{A}))$ is a limit of Borel functions, and is itself Borel.

THEOREM 2.8. $\mathscr{F}_{\text {fin }}$ is a Borel subset of $\mathscr{F}$.

Proof. Choose $\mathfrak{A} \rightarrow U_{i}(\mathfrak{H})$ and $\mathfrak{A} \rightarrow E(\mathfrak{A})$ as in Lemmas 2.6 and 2.7. Let $d$ be a metric for the weak topology on $\mathfrak{L}_{\infty, 1}$. If $\mathfrak{A} \in \mathscr{A}_{\infty}$ and $0 \in \kappa_{\mathfrak{A}}(E(\mathfrak{H})$ ), then given $\varepsilon>0$, there exist finitely many unitaries $V_{j} \in \mathfrak{A}$ and non-negative reals $t_{j}$ with $\Sigma t_{j}=1$ and

$$
d\left(\sum t_{j} V_{j} E(\mathfrak{X}) V_{j}^{*}, 0\right)<\varepsilon .
$$

As multiplication is strongly continuous on bounded sets, and the adjoint operation is strongly continuous on the unitaries, we may choose non-negative rationals $r_{i}$ with $\sum r_{i}=1$ and

Letting

$$
d\left(\sum r_{i} U_{i}(\mathfrak{A}) E(\mathfrak{A}) U_{i}(\mathfrak{A})^{*}, 0\right)<\varepsilon .
$$

$$
\Delta_{1}=\left\{\mathfrak{A} \in \mathscr{A}_{\infty}: \inf d\left(\sum r_{i} U_{i}(\mathfrak{A}) E(\mathfrak{A}) U_{i}(\mathfrak{A})^{*}, 0\right)=0\right\},
$$

the inf being taken over all such finite rational convex sums, $\Delta_{1}$ is Borel, and consists of those $\mathfrak{A} \in \mathscr{A}_{\infty}$ for which $0 \in \kappa_{\mathfrak{A}}(E(\mathfrak{Q}))$. Similarly the set $\Delta_{2}$ of $\mathfrak{A} \in \mathscr{A}_{\infty}$ with $0 \in \kappa_{\mathfrak{A}}(I-E(\mathfrak{Q}))$ is Borel. From Lemma 2.5, 


$$
\mathscr{F}_{\text {fin }}=\left(\bigcup_{n<\infty} \mathscr{F}_{n}\right) \cup\left[\mathscr{F}_{\infty}-\left(\Delta_{1} \cup \Delta_{2}\right)\right] \cup\left\{\mathfrak{J}_{\infty}\right\}
$$

and $\mathscr{F}_{\text {fin }}$ is Borel.

3. Coupling and the index spaces. If $x$ is a vector in $\mathfrak{H}_{n}$ and $\mathfrak{U} \in \mathscr{A}_{n}$, let $[\mathfrak{U} x] \in \mathfrak{U}^{\prime}$ be the projection on the smallest linear space containing $x$, invariant under $\mathfrak{A}$. If $\mathfrak{A} \in \mathscr{F}_{\text {fin }}$, let $\tau_{\mathfrak{U}}$ be the normal trace on $\mathfrak{U}$ with $\tau_{\mathfrak{A}}(I)=1$. If $\mathfrak{U} \in \mathscr{F}_{\text {fin }} \cap \mathscr{F}_{\text {fin }}^{\prime}$, and $x$ is a nonzero vector in the underlying Hilbert space, $\tau_{\mathfrak{H}}\left(\left[\mathfrak{U}^{\prime} x\right]\right) / \tau_{\mathfrak{H}}([\mathfrak{U} x])$ does not depend on $x$, and is known as the coupling $C(\mathfrak{U})$ of $\mathfrak{A}$. Two algebraically isomorphic algebras in $\mathscr{F}_{\text {fin }}^{\prime} \cap \mathscr{F}_{\text {fin }}$ are spatially isomorphic if and only if they have the same coupling (see [2, Chapter III, §6.4]).

LEMMA 3.1. If $x \in \mathfrak{H}_{n}, \mathfrak{U} \rightarrow[\mathfrak{U} x]$ is a Borel map of $\mathscr{A}_{n}$ into $\mathfrak{Q}_{n}$.

Proof. If $E \in \mathfrak{L}_{n}$ is a projection and $y \in \mathfrak{H}_{n}$,

$$
\|(I-E) y\|=\inf \left\{\|y-z\|: z \in E \mathfrak{S}_{n}\right\} \text {. }
$$

This is due to the fact that the closest vector in $E \mathfrak{H}_{n}$ to $y$ is $E y$ (see $\left.[11, \S 11]\right)$. Let $A_{i}(\mathfrak{A})$ be Borel choice functions on $\mathscr{A}_{n}$, weakly dense in $\mathfrak{A}_{1}$ for each $\mathfrak{A}$, and $B_{j}(\mathfrak{U})$ be the finite rational combinations of the $A_{i}(\mathfrak{U})$. The strong closure of the $B_{j}(\mathfrak{U})$ is linear, hence weakly closed, and contains $\mathfrak{U}_{1}$. Thus the $B_{j}(\mathfrak{U})$ are strongly dense in $\mathfrak{A}$, and for $y \in \mathfrak{H}_{n}$,

$$
\|(I-[\mathfrak{A} x]) y\|=\inf \left\{\left\|y-B_{j}(\mathfrak{U}) x\right\|\right\} .
$$

Thus

$$
\mathfrak{U} \rightarrow[\mathfrak{A} x] y \cdot y=\|y\|^{2}-\|(I-[\mathfrak{A} x]) y\|^{2}
$$

is Borel, and the lemma follows.

LEMMA 3.2. If $\mathfrak{A} \rightarrow A(\mathfrak{U}) \in \mathfrak{A}_{1}$ is a Borel choice function on $\mathscr{F}_{\text {fin }}$, then $\mathfrak{A} \rightarrow \tau_{\mathfrak{H}}(A(\mathfrak{A}))$ is also Borel.

Proof. It suffices to prove that the function is Borel on $\mathscr{F}_{\text {fin }} \cap \mathscr{A}_{n}$. From Lemma 2.6, we may let $\mathfrak{A} \rightarrow U_{i}(\mathfrak{A})$ be Borel with $U_{i}(\mathfrak{U})$ strongly dense in the unitaries of $\mathfrak{A}$. Let $d$ be a metric for the weak topology on $\mathfrak{L}_{n, 1}$. Fixing $\mathfrak{A}$, $\tau_{\mathfrak{U}}(A(\mathfrak{U})) I$ is the unique element in $\kappa_{\mathfrak{A}}(A(\mathfrak{U})) \cap \mathfrak{I}_{n}[2$, p. 272]. Thus given a closed subset $F$ of the complex plane, if $\tau_{\mathfrak{H}}(A(\mathfrak{U})) \in F$, then for any $\varepsilon>0$ there exist non-negative rationals $r_{i}$ with $\Sigma r_{i}=1$ and

$$
d\left(\sum r_{i} U_{i}(\mathfrak{Q}) A(\mathfrak{Q}) U_{i}(\mathfrak{A})^{*}, F I\right) \leqq \varepsilon .
$$

Conversely if for each integer $j$ there is a finite rational convex sum $B_{j}$ satisfying the above inequality for $\varepsilon=1 / j$, let $B_{j_{k}}$ be a weakly convergent subsequence. We have $\tau_{\mathfrak{U}}(A(\mathfrak{U})) I=\lim B_{j_{k}} \in F I$. Letting $T(\mathfrak{P})=\tau_{\mathfrak{U}}(A(\mathfrak{U}))$, 


$$
T^{-1}(F)=\left\{\mathfrak{A}: \inf d\left(\sum r_{i} U_{i}(\mathfrak{U}) A(\mathfrak{U}) U_{i}(\mathfrak{U})^{*}, F I\right)=0\right\},
$$

the inf taken over all finite convex rational sums, hence $T^{-1}(F)$, and $T$, are Borel.

THEOREM 3.3. The coupling function $\mathfrak{A} \rightarrow C(\mathfrak{A})$ on $\mathscr{F}_{\text {fin }} \cap \mathscr{F}_{\text {fin }}^{\prime}$ is Borel.

Proof. Let $x_{n}$ be a fixed nonzero vector in $\mathfrak{H}_{n}$. As $\mathfrak{A} \rightarrow \mathfrak{A}^{\prime}$ is Borel, we have from the above lemmas that

is Borel on $\mathscr{F}_{\text {fin }} \cap \mathscr{F}_{\text {fin }}^{\prime} \cap \mathfrak{A}_{n}$.

$$
\mathfrak{U} \rightarrow C(\mathfrak{A})=\tau_{\mathfrak{Q}}\left(\left[\mathfrak{U}^{\prime} x_{n}\right]\right) / \tau_{\mathfrak{A}},\left(\left[\mathfrak{A} x_{n}\right]\right)
$$

From Corollary 2.3, Borel sets in $\hat{\mathscr{F}}$ and $\tilde{\mathscr{F}}$ separate points. We recall that a Borel space is countably separated if there exists a countable family of Borel sets, or equivalently, complex-valued Borel functions, separating points. As $\mathscr{F}$ is standard, should $\hat{\mathscr{F}}$ or $\tilde{\mathscr{F}}$ be countably separated, it would be analytic [18, p. 141].

THEOREM 3.4. $\hat{\mathscr{F}}$ is countably separated if and only if $\tilde{\mathscr{F}}$ is countably separated.

Proof. Let $\pi_{1}$ and $\pi_{2}$ be the quotient maps of $\mathscr{F}$ onto $\hat{\mathscr{F}}$ and $\tilde{\mathscr{F}}$, respectively, and define $\alpha$ by $\alpha \circ \pi_{1}=\pi_{2} . \alpha$ is Borel, as if $B$ is Borel in $\tilde{\mathscr{F}}, \pi_{1}^{-1}\left(\alpha^{-1}(B)\right)=\pi_{2}^{-1}(B)$ is Borel in $\mathscr{F}$. Extend coupling to $\mathscr{F}$ by letting $C(\mathfrak{A})=0$ for $\mathfrak{A} \notin \mathscr{F}_{\text {fin }} \cap \mathscr{F}_{\text {fin }}^{\prime}$. $C$ is Borel (Theorems 2.8 and 3.3), and constant on spatial equivalence classes, hence it defines a Borel function $\hat{C}$ on $\hat{F}$. Similarly the commutant operation ' defines a Borel isomorphism on $\hat{\mathscr{F}}$, which we again indicate by '. Let $\mathscr{F}_{\text {inf }}$ be the infinite factors.

Suppose there exist Borel functions $f_{i}, i=1,2, \cdots$, separating points in $\tilde{\mathscr{F}}$. Then we claim the functions $f_{i} \circ \alpha, f_{i} \circ \alpha \circ \circ^{\prime}$, and $\hat{C}$ together separate points in $\hat{F}$. As two factors with infinite commutants are spatially isomorphic if and only if they are algebraically isomorphic, the $f_{i} \circ \alpha$ separate points in $\hat{\mathscr{F}}_{\text {inf }}^{\prime}$. It follows that the $f_{i} \circ \alpha \circ^{\prime}$ separate points in $\hat{\mathscr{F}}_{\text {inf }} . \hat{C}$ together with the $f_{i} \circ \alpha$ separate points in $\hat{\mathscr{F}}_{\text {fin }} \cap \hat{\mathscr{F}}_{\text {fin }}^{\prime}$. The $f_{i} \circ \alpha$ separate $\hat{\mathscr{F}}_{\text {fin }}$ from $\hat{\mathscr{F}}_{\text {inf }}$, hence all points are separated.

Let $T$ be the Borel set $\left\{\mathfrak{U} \in \mathscr{F}_{\text {fin }} \cap \mathscr{F}_{\text {fin }}^{\prime}: C(\mathfrak{U})=1\right\} \cup\left[\mathscr{F}_{\text {inf }} \cap \mathscr{F}_{\text {inf }}^{\prime}\right]$, and $\hat{T}=\pi_{1}(T) . \alpha$ is a one-to-one Borel map of $\hat{T}$ onto $\tilde{\mathscr{F}}$. We shall prove that $\alpha$ is a Borel isomorphism on $\hat{T}$. It will follow that if $\hat{\mathscr{F}}$ is countably separated, then so is $\tilde{\mathscr{F}}$.

If $B$ is a subset of $\mathscr{F}$, let $B^{s}$ and $B^{a}$ be the saturations of $B$ with respect to spatial and algebraic equivalence, respectively. Using the notation of $\S 2$, if $B$ is Borel

$$
B^{s}=\bigcup_{\phi_{n}}\left(G_{n} \times\left(B \cap \mathscr{A}_{n}\right)\right)
$$

and

$$
B^{a}=\theta^{-1}\left(\theta(B)^{s}\right)
$$

are analytic. If $D$ is a Borel subset of $\hat{T}$, 
and

$$
\pi_{2}^{-1}(\alpha(D))=\left(\pi_{1}^{-1}(D)\right)^{a}
$$

$$
\begin{aligned}
\pi_{2}^{-1}(\tilde{\mathscr{F}}-\alpha(D)) & =\pi_{2}^{-1}(\alpha(\hat{T}-D)) \\
& =\left(\pi_{1}^{-1}(\hat{T}-D)\right)^{a}
\end{aligned}
$$

are disjoint analytic sets. As their union is the standard space $\mathscr{F}$, both must be Borel (see $[13, \S 35$, III $]$ ), hence $\alpha(D)$ is Borel in $\tilde{\mathscr{F}}$.

4. Direct integral theory. We begin with a brief summary of the constructive theory in order to introduce our terminology. Details and omitted proofs may be found in [2, Chapter II].

As suggested in [7, pp. 83-84] and [19, p. 634], one may regard a field of objects over a Borel space as a cross-section in an appropriate "bundle". We shall instead use "coherences" to map fields into bundles with constant fiber. (We essentially follow [17].) It is then unnecessary to introduce the bundle terminology.

By a measure $\mu$ on a Borel space $(Z, \mathscr{S})$, we mean a real, non-negative, finite, countably additive function on $\mathscr{S}$. If $x: Z \rightarrow \mathfrak{H}_{n}$ is weakly Borel, let $x(\mu)$ be the class of weakly Borel functions of $Z$ into $\mathfrak{H}_{n}$, equal to $x \mu$-almost everywhere. Let $\bar{L}_{n}^{2}(\mu)=\bar{L}_{n}^{2}(Z, \mathscr{S}, \mu)$ be the weakly Borel functions $x: Z \rightarrow \mathfrak{S}_{n}$ with

$$
\int\|x(\zeta)\|^{2} d \mu(\zeta)<\infty
$$

$\bar{L}_{n}^{2}(\mu)$ is a linear space with Hilbert pseudo-norm $\|x\|=\left[\int\|x(\zeta)\|^{2} d \mu(\zeta)\right]^{1 / 2}$, and null space the functions equal to $0 \mu$-almost everywhere. Let $L_{n}^{2}(\mu)=L_{n}^{2}(Z, \mathscr{S}, \mu)$ be the quotient Hilbert space.

If $A: Z \rightarrow \mathfrak{Q}_{n}$ is a uniformly bounded, weakly Borel function, define $\bar{A}$ on $\bar{L}_{n}^{2}(\mu)$ by $(\bar{A}(x))(\zeta)=A(\zeta) x(\zeta) . \bar{A}$ preserves null functions, and induces a map $A(\mu)$ on $L_{n}^{2}(\mu)$ with $\|A(\mu)\|=$ ess. sup $\|A(\zeta)\|$. If $\mathfrak{A}: Z \rightarrow \mathscr{A}_{n}$ is Borel, let $\mathfrak{A}(\mu)=\int \mathfrak{A}(\zeta) d \mu(\zeta)$ be all operators of the form $A(\mu)$, where $A: Z \rightarrow \mathfrak{L}_{n}$ is a uniformly bounded weakly Borel function with $A(\zeta) \in \mathfrak{U}(\zeta)$ for all $\zeta . \mathfrak{A}(\mu)$ is a von Neumann algebra with $\mathfrak{U}(\mu)^{\prime}=\mathfrak{U}^{\prime}(\mu)$.

Let $\mathfrak{S}_{0}=\mathfrak{L}_{0}=\mathscr{A}_{0}=\{0\}$. As it will be necessary to consider four varieties of fields, we introduce the "fibers":

$$
\begin{aligned}
\mathscr{H} & =\left\{\mathfrak{H}_{0}, \mathfrak{H}_{1}, \cdots, \mathfrak{H}_{\infty}\right\}, \\
\mathfrak{H}_{u} & =\bigcup_{n=0}^{\infty} \mathfrak{H}_{n}, \\
\mathfrak{L}_{u} & =\bigcup_{n=0}^{\infty} \mathfrak{L}_{n}, \\
\mathscr{A}_{u} & =\bigcup_{n=0}^{\infty} \mathscr{A}_{n}=\mathscr{A} \cup \mathscr{A}_{0},
\end{aligned}
$$


where we regard the last three unions as disjoint. Let $\mathscr{H}$ have the discrete Borel structure, $\mathfrak{H}_{u}$ and $\mathfrak{L}_{u}$ have, the structures generated by the weak (or, equivalently, strong) structures on the $\mathfrak{S}_{n}$ and $\mathfrak{L}_{n}$ and $\mathscr{A}_{u}$ have the structure generated by those on the $\mathscr{A}_{n}$.

If $\mathfrak{H}: Z \rightarrow \mathscr{H}$ is Borel and $Z_{n}=\left\{\zeta: \mathfrak{H}(\zeta)=\mathfrak{H}_{n}\right\}$, define $\mathfrak{H}(\mu)=\int \mathfrak{H}(\zeta) d \mu(\zeta)$ to be $\sum_{n=1}^{\infty} L_{n}^{2}\left(\mu_{n}\right)$, where $\mu_{n}$ is the restriction of $\mu$ to $Z_{n} \cdot \mathfrak{H}(\mu)$ consists of all sequences $x(\mu)=\left(x\left(\mu_{n}\right)\right)$, where $x: Z \rightarrow \mathfrak{Y}_{u}$ is Borel with $x(\zeta) \in \mathfrak{H}_{n}$ for $\zeta \in Z_{n}$ and

$$
\|x(\mu)\|^{2}=\sum_{n=1}^{\infty} \int\|x(\zeta)\|^{2} d \mu_{n}(\zeta)=\int\|x(\zeta)\|^{2} d \mu(\zeta)<\infty .
$$

Similarly, if $\mathfrak{A}: Z \rightarrow \mathscr{A}_{u}$ is Borel, and $Z_{n}=\left\{\zeta: \mathfrak{U}(\zeta) \in \mathscr{A}_{n}\right\}$, we define $\mathfrak{A}(\mu)=\int \mathfrak{A}(\zeta) d \mu(\zeta)$ to be $\sum_{n=1}^{\infty} \mathfrak{A}\left(\mu_{n}\right)$. Assuming the $Z_{n}$ for $\mathfrak{H}$ and $\mathfrak{A}$ coincide, $\mathfrak{A}(\mu)$ is defined on $\mathfrak{H}(\mu)$. It consists of all sequences $A(\mu)=\left(A\left(\mu_{n}\right)\right)$, where $A: Z \rightarrow \mathfrak{L}_{u}$ is uniformly bounded and Borel, and $A(\zeta) \in \mathfrak{A}(\zeta)$ for all $\zeta$.

If $Z$ is a set, a field of Hilbert spaces $\mathfrak{H}$ on $Z$ is a $\operatorname{map} \zeta \rightarrow \mathfrak{H}(\zeta)$ of $Z$ into a collection of separable Hilbert spaces. Letting $Z_{n}=\{\zeta: \operatorname{dim} \mathfrak{S}(\zeta)=n\}$ a coherence $\gamma$ for $\mathfrak{S}$ is a map $\zeta \rightarrow \gamma(\zeta)$, where $\gamma(\zeta)$ is an isometry of $\mathfrak{Y}(\zeta)$ onto $\mathfrak{S}_{n}$ for $\zeta \in Z_{n}$. A vector field $x$ in $\mathfrak{S}$ is a map $\zeta \rightarrow x(\zeta) \in \mathfrak{S}(\zeta)$, an operator field $A$ on $\mathfrak{S}$ is a map $\zeta \rightarrow A(\zeta) \in \mathfrak{L}(\mathfrak{S}(\zeta))$, and a field of von Neumann algebras $\mathfrak{A}$ on $\mathfrak{H}$ is a map $\zeta \rightarrow \mathfrak{A}(\zeta) \in \mathscr{A}(\mathfrak{T}(\zeta))$. If $Z$ has a Borel structure and $\gamma$ is a coherence for $\mathfrak{H}$, we say these fields are $\gamma$-Borel when

$$
\begin{aligned}
\mathfrak{S}^{\gamma}(\zeta) & =\gamma(\zeta) \mathfrak{S}(\zeta), \\
x^{\gamma}(\zeta) & =\gamma(\zeta) x(\zeta), \\
A^{\gamma}(\zeta) & =\gamma(\zeta) A(\zeta) \gamma(\zeta)^{-1}, \\
\mathfrak{A}^{\gamma}(\zeta) & =\gamma(\zeta) \mathfrak{A}(\zeta) \gamma(\zeta)^{-1},
\end{aligned}
$$

are Borel maps of $Z$ into $\mathscr{H}, \mathfrak{H}_{u}, \mathfrak{L}_{u}$, and $\mathscr{A}_{u}$, respectively. As $\mathfrak{H}$ is $\gamma$-Borel if and only if the sets $Z_{n}$ are Borel, the coherence is irrelevant, and we say $\mathfrak{H}$ is Borel if the latter is true. If $\mu$ is a measure on $Z$, we write $\int^{\gamma} \mathfrak{H}(\zeta) d \mu(\zeta)$ and $1 \int^{\gamma} \mathfrak{A}(\zeta) d \mu(\zeta)$ for $\int \mathfrak{S}^{\gamma}(\zeta) d \mu(\zeta)$ and $\int \mathfrak{U}^{\gamma}(\zeta) d \mu(\zeta)$, respectively.

Let $I$ be a finite or countable set. For each sequence $m=\left(m_{i}\right)_{i \in I}$ of non-negative integers, let $\sigma_{m}$ be an isometry of $\Sigma \mathfrak{S}_{m_{i}}$ onto $\mathfrak{H}_{\Sigma m_{i}}$. If $\left(\mathfrak{S}^{i}, \gamma_{i}\right)$ are Borel coherent fields (i.e., each $\mathfrak{S}^{i}$ is Borel and has coherence $\gamma_{i}$ ), and $\zeta$ is such that $\operatorname{dim} \mathfrak{S}^{i}(\zeta)=m_{i}$, define for $\left(x_{i}\right) \in \sum \mathfrak{S}^{i}(\zeta)$

$$
\left(\Sigma_{\gamma_{i}}(\zeta)\right)\left(x_{i}\right)=\sigma_{m}\left(\gamma_{i}(\zeta) x_{i}\right)
$$

Then $\left(\Sigma \mathfrak{S}_{i}, \Sigma \gamma_{i}\right)$ is a Borel coherent field. If $\mathfrak{U}_{i}$ are $\gamma_{i}$-Borel, then $\Sigma \mathfrak{U}_{i}$ is $\Sigma \gamma_{i^{-}}$ Borel, and

$$
\int^{\Sigma \gamma_{t}} \Sigma \mathfrak{A}_{i}(\zeta) d \mu(\zeta) \cong \Sigma \int^{\gamma_{t}} \mathfrak{A}_{i}(\zeta) d \mu(\zeta)
$$


Without going into details (see the proof of Lemma 4.5), we remark that the underlying isometry is defined by

$$
\left(x_{i}\right)^{\Sigma \gamma_{i}}(\mu) \rightarrow\left(x_{i}^{\gamma_{i}}(\mu)\right)
$$

where $x_{i}$ is a sequence of $\gamma_{i}$-Borel vector fields with $\sum \int\left\|x_{i}\right\|^{2} d \mu<\infty$.

Let $\tau_{m, n}$ be a fixed isometry of $\mathfrak{H}_{m} \otimes \mathfrak{Y}_{n}$ onto $\mathfrak{H}_{m n}$ for $m, n \geqq 0$ (we let $0 \cdot \infty=0$ ). If $(\mathfrak{H}, \gamma)$ and $(\boldsymbol{R}, \delta)$ are Borel coherent fields, and $\zeta$ is such that $\operatorname{dim} \mathfrak{H}(\zeta)=m$, $\operatorname{dim} \mathfrak{R}(\zeta)=n$, let $\gamma(\zeta) \otimes \delta(\zeta)$ be the unique linear isometry of $\mathfrak{H}(\zeta) \otimes \mathfrak{R}(\zeta)$ onto $\mathfrak{H}_{m} \otimes \mathfrak{H}_{n}$ satisfying for $u \in \mathfrak{H}(\zeta), v \in \mathfrak{R}(\zeta), \gamma(\zeta) \otimes \delta(\zeta)(u \otimes v)=\gamma(\zeta)(u) \otimes \delta(\zeta)(v)$. Let $\gamma \otimes \delta(\zeta)=\tau_{m, n} \circ(\gamma(\zeta) \otimes \delta(\zeta))$. Then $(\mathfrak{H} \otimes \mathfrak{H}, \gamma \otimes \delta)$ is a Borel coherent field. If $\mathfrak{A}$ and $\mathfrak{B}$ are $\gamma$ and $\delta$-Borel respectively, $\mathfrak{A} \otimes \mathfrak{B}$ is $\gamma \otimes \delta$-Borel. If $\mathfrak{B}^{\delta}(\zeta)=\mathfrak{B}_{0}$ for all $\zeta$,

$$
\int^{\gamma \otimes \boldsymbol{d}} \mathfrak{A} \otimes \mathfrak{B}(\zeta) d \mu(\zeta) \cong \int^{\gamma} \mathfrak{U}(\zeta) d \mu(\zeta) \otimes \mathfrak{B}_{0}
$$

Let $\mathfrak{S}_{n}$ underlie $\mathfrak{B}_{0}, v_{j}$ be arbitrary vectors in $\mathfrak{Y}_{n}$, and $y_{j}(\zeta)=\delta(\zeta)^{-1}\left(v_{j}\right)$. The spatial isometry of (2) is the closure of the map

$$
\Sigma\left(x_{i} \otimes y_{j}\right)^{\gamma \otimes \delta}(\mu) \rightarrow \sum x_{i}^{\gamma}(\mu) \otimes v_{j},
$$

where the sums are finite, and the $x_{i}$ are $\gamma$-Borel vector fields with $\int\left\|x_{i}\right\|^{2} d \mu<\infty$ If $\mathfrak{A}(\zeta)=\mathfrak{Q}_{1}$ (the complex numbers) for all $\zeta$, it follows that

$$
\int \mathfrak{B}_{0} d \mu(\zeta) \cong L^{\infty}(\mu) \otimes \mathfrak{B}_{0},
$$

where $L^{\infty}(\mu)$ acts on $L^{2}(\mu)$ by multiplication.

Suppose that $Z_{1}$ and $Z_{2}$ are Borel spaces with measure $\mu_{1}$ and $\mu_{2}$ respectively. A measure isomorphism $\left(T, N_{1}, N_{2}\right)$ of $Z_{1}$ and $Z_{2}$ is a Borel isomorphism $T$ of $Z_{1}-N_{1}$ onto $Z_{2}-N_{2}$, where $N_{i}$ are Borel in $Z_{i}, \mu_{i}\left(N_{i}\right)=0$, such that the $\mu_{2}$ null sets in $Z_{2}-N_{2}$ are the images of the $\mu_{1}$-null sets in $Z_{1}-N_{1}$.

LEMMA 4.1. Say that $Z_{i}, i=1,2$ are standard Borel spaces with Borel coherent fields $\left(\mathfrak{S}_{i}, \gamma_{i}\right), \gamma_{i}$-Borel fields $\mathfrak{U}_{i}$, and measures $\mu_{i}$. If there is a measure isomorphism $\left(T, N_{1}, N_{2}\right)$ of $Z_{1}$ and $Z_{2}$ with $\mathfrak{A}_{1}\left(\zeta_{1}\right) \cong \mathfrak{A}_{2}\left(T\left(\zeta_{1}\right)\right)$ for all $\zeta \in Z_{1}-N_{1}$, then $\int^{\gamma_{1}} \mathfrak{U}_{1}\left(\zeta_{1}\right) d \mu_{1}\left(\zeta_{1}\right) \cong \int^{\gamma_{2}} \mathfrak{U}_{2}\left(\zeta_{2}\right) d \mu_{2}\left(\zeta_{2}\right)$.

We say a family of vectors $\left\{x_{\alpha}\right\}$ spans a topological vector space $\mathfrak{X}$ if the linear space generated by the $x_{\alpha}$ is dense in $\mathfrak{X}$. Rephrasing some of the results in [2, Chapter II], we have

LEMMA 4.2. Suppose that $(\mathfrak{H}, \gamma)$ is a Borel coherent field on a Borel space $Z$. Given a measure $\mu$ on $Z$, say $g_{i}$ are bounded Borel functions with $g_{i}(\mu)$ spanning $L^{\infty}(\mu)$ in the weak ${ }^{*}$ topology. If $x_{j}$ is a sequence of uniformly bounded $\gamma$-Borel vector fields in $\mathfrak{H}$ with $x_{j}(\zeta)$ spanning $\mathfrak{H}(\zeta)$ for all $\zeta$, then the vectors $g_{i} x_{j}^{\gamma}(\mu)$ 
span $\mathfrak{H}^{\gamma}(\mu)$. If $\mathfrak{A}$ is a $\gamma$-Borel field of von Neumann algebras on $Z$, and $A_{k}$ is a sequence of uniformly bounded $\gamma$-Borel operator fields in $\mathfrak{A}$, with $A_{k}(\zeta)$, generating $\mathfrak{A}(\zeta)$, the operators $A_{k}^{\gamma}(\mu)$, together with the $g_{i}(\mu) I$, generate $\mathfrak{A}^{\gamma}(\mu)$.

Proof. Let $x$ be a $\gamma$-Borel field in $\mathfrak{H}$ with $x^{\gamma}(\mu) \in \mathfrak{S}^{\gamma}(\mu)$, and $x^{\gamma}(\mu) \perp g_{i} x_{j}^{\gamma}(\mu)$ for all $i, j$. Then

$$
\begin{aligned}
0 & =x^{\gamma}(\mu) \cdot g_{i} x_{j}^{\gamma}(\mu) \\
& =\sum_{n} \int x^{\gamma}(\zeta) \cdot g_{i}(\zeta) x_{j}^{\gamma}(\zeta) d \mu_{n}(\zeta) \\
& =\sum_{n} \int\left[x(\zeta) \cdot x_{j}(\zeta)\right] \bar{g}_{i}(\zeta) d \mu_{n}(\zeta) \\
& =\int\left[x(\zeta) \cdot x_{j}(\zeta)\right] \bar{g}_{i}(\zeta) d \mu(\zeta)
\end{aligned}
$$

for all $i$ implies $x(\zeta) \cdot x_{j}(\zeta)=0$ a.e. It follows that $x(\zeta)=0$ a.e., hence $x(\mu)=0$.

Let $\mathfrak{B}$ be the von Neumann algebra generated by the $A_{k}^{\gamma}(\mu)$ and $g_{i}(\mu) I$. It suffices to prove that $\mathfrak{B}^{\prime} \subseteq \mathfrak{A}^{\gamma}(\mu)^{\prime}$. If $B^{\prime} \in \mathfrak{B}^{\prime}, B^{\prime}$ commutes with the $g_{i}(\mu) I$. From the decomposition theory, it follows that there is a uniformly bounded $\gamma$-Borel operator field $\zeta \rightarrow C(\zeta) \in \mathfrak{L}(\mathfrak{H}(\zeta))$ with $B^{\prime}=C^{\gamma}(\mu)$. As $B^{\prime}$ commutes with $A_{k}(\mu), C(\zeta)$ commutes with $A_{k}(\zeta)$ a.e., hence $C(\zeta) \in \mathfrak{A}(\zeta)^{\prime}$ a.e., and $B^{\prime} \in \mathfrak{A}^{\prime}(\mu)=\mathfrak{A}(\mu)^{\prime}$.

If $Z$ is a Borel space, let $M(Z)$ be the measures on $Z$, together with the Borel structure defined by the functions $\mu \rightarrow \int f d \mu$, with $f$ a bounded Borel function on $Z$. If $Z$ is standard, let $Z$ have a compact metrizable topology generating its structure, and let $\mathfrak{C}(Z)$ be the continuous functions. $M(Z)$ is Borel isomorphic to the positive cone of $\mathfrak{C}(Z)^{*}$ with the weak* topology, and thus is standard.

Lemma 4.3. Say that $Z$ is standard, and $\mathfrak{H}: Z \rightarrow \mathscr{H}$ is Borel. Then $\mu \rightarrow \mathfrak{S}(\mu)$ is a Borel field on $M(Z)$, and there exists a coherence $\gamma(\mu)$ for $\mathfrak{G}(\mu)$ with the following properties:

(1) If $x: Z \rightarrow \mathfrak{H}_{u}$ is a uniformly bounded Borel field in $\mathfrak{H}, \mu \rightarrow x(\mu)$ is a $\gamma$ Borel field in $\mu \rightarrow \mathfrak{S}(\mu)$, uniformly bounded on $M(Z)_{1}$.

(2) If $A: Z \rightarrow \mathfrak{L}_{u}$ is a uniformly bounded Borel field on $\mathfrak{H}, \mu \rightarrow A(\mu)$ is a uniformly bounded $\gamma$-Borel field on $\mu \rightarrow \mathfrak{S}(\mu)$.

(3) If $\mathfrak{A}: Z \rightarrow \mathscr{A}_{u}$ is a Borel field on $\mathfrak{H}, \mu \rightarrow \mathfrak{A}(\mu)$ is a $\gamma$-Borel field in $\mu \rightarrow \mathfrak{H}(\mu)$.

Proof. Let $Z$ have a compact metrizable topology generating its structure, and let $\mathfrak{C}(Z)$ be the continuous functions on $Z$. Choose $g_{i}$ uniformly dense in $\mathfrak{C}(Z)$. Then for each $\mu \in M(Z)$, the $g_{i}(\mu)$ span $L^{\infty}(\mu)$ in the weak* topology. Let $e_{n_{1}}, e_{n_{2}}, \cdots$ be an orthonormal basis for $\mathfrak{H}_{n}, n \geqq 1$, and define $x_{n j}(\zeta)=e_{n j}$ for $\zeta \in Z_{n}, x_{n j}(\zeta)=0$ elsewhere. From Lemma 4.2, the vectors $g_{i} x_{n j}(\mu)$ span $\mathfrak{S}(\mu)$. Furthermore, for any combination of subscripts, 


$$
\mu \rightarrow g_{i} x_{n j}(\mu) \cdot g_{i^{\prime}} x_{n^{\prime} j}(\mu)= \begin{cases}\int g_{i} \bar{g}_{i}, d \mu & \text { it } n=n^{\prime}, j=j^{\prime}, \\ 0 & \text { otherwise, }\end{cases}
$$

is Borel on $M(Z)$. Let $v_{p}(\zeta)$ be an enumeration of the fields $g_{i}(\zeta) x_{n j}(\zeta)$. A simultaneous orthonormalization procedure (see [2, p. 139]) on the $v_{p}(\mu), \mu \in M(Z)$, yields vector fields

$$
w_{k}(\mu)=\sum_{1 \leqq p \leqq k} h_{p}^{k}(\mu) v_{p}(\mu)
$$

where $h_{p}^{k}(\mu)$ is a Borel scalar function on $M(Z)$, and the $w_{k}(\mu), k \leqq \operatorname{dim} \mathfrak{S}(\mu)$ form an orthonormal basis for $\mathfrak{H}(\mu), w_{k}(\mu)=0$ for $k>\operatorname{dim} \mathfrak{S}(\mu)$. From (5) and (4), $\mu \rightarrow\left\|w_{k}(\mu)\right\|^{2}$ is Borel, hence the set $M_{m}$ of $\mu$ with $\operatorname{dim} \mathfrak{H}(\mu)=m$ is Borel, and $\mu \rightarrow \mathfrak{S}(\mu)$ is Borel. If $\mu \in M_{m}$, define $\gamma(\mu): \mathfrak{S}(\mu) \rightarrow \mathfrak{S}_{m}$ by

$$
\gamma(\mu) y=\sum_{k}\left(y \cdot w_{k}(\mu)\right) e_{m k} .
$$

A vector field $\mu \rightarrow y(\mu)$ will be $\gamma$-Borel if and only if $y(\mu) \cdot w_{k}(\mu)$ is Borel for all $k$, hence from (5), if and only if $y(\mu) \cdot g_{i} x_{n j}(\mu)$ are all Borel.

Let $x, A$, and $\mathfrak{A}$ be as described above. Since

$$
\mu \rightarrow x(\mu) \cdot g_{i} x_{n j}(\mu)=\sum_{n} \int_{Z_{n}} x(\zeta) \cdot e_{n j} \bar{g}_{i}(\zeta) d \mu(\zeta)
$$

is Borel, $\mu \rightarrow x(\mu)$ is $\gamma$-Borel, and if $\|x(\zeta)\| \leqq K,\|x(\mu)\| \leqq K \mu(Z)^{1 / 2}$. Turning to $A$, if $\|A(\zeta)\| \leqq L,\|A(\mu)\| \leqq L$. To show that $\mu \rightarrow A^{\gamma}(\mu)$ is Borel, it suffices to prove that on $M_{m}$

$$
\mu \rightarrow A^{\gamma}(\mu) e_{m i} \cdot e_{m j}=A(\mu) w_{i}(\mu) \cdot w_{j}(\mu)
$$

is Borel. This follows from (5) as

$$
\begin{aligned}
\mu & \rightarrow A(\mu) g_{i} x_{n j}(\mu) \cdot g_{i^{\prime}} x_{n j}(\mu) \\
& =\int_{Z_{n}}\left(A(\zeta) e_{n j} \cdot e_{n j^{\prime}}\right) g_{i}(\zeta) \bar{g}_{i^{\prime}}(\zeta) d \mu(\zeta)
\end{aligned}
$$

is Borel.

From [5], we may select uniformly bounded Borel fields $A_{j}(\zeta)$ in $\mathfrak{U}(\zeta)$ generating $\mathfrak{A}(\zeta)$ at each $\zeta$. From Lemma 4.2 , the fields $A_{j}^{\gamma}(\mu)$ and $g_{i}(\mu) I$ generate $\mathfrak{A}^{\gamma}(\mu)$ at each $\mu$, hence $\mu \rightarrow \mathfrak{U}^{\gamma}(\mu)$ is a Borel map of $M(Z)$ into $\mathscr{A}_{u}$ (see discussion at the end of $\$ 3$ in [5]).

Let $R$ be an equivalence relation on a Borel space $X$, and $\pi$ be the quotient map of $X$ onto the set of equivalence classes $X / R$. Providing $X / R$ with the quotient structure, we say that $R$ is smooth if $X / R$ is countably separated. If $\mu$ is a measure on $X$, define the quotient measure $v$ on $X / R$ by $v(T)=\mu\left(\pi^{-1}(T)\right)$. We have 
included the following version of the decomposition theorem for $\mu$ (see [1], $[21],[9],[20])$ as it gives partial results for non smooth relations.

LEMMA 4.4. Suppose that $R$ is an equivalence relation on a standard Borel space $Z, \mu$ is a measure on $Z$, and $v$ is the quotient measure on $Z / R$. Then there exists a Borel map $\xi \rightarrow \mu_{\xi}$ of $Z / R$ into $M(Z)_{1}$ such that if $f$ is a bounded Borel function on $Z$, and $h$ is a $v$-integrable function on $Z / R$, then

$$
\int h \circ \pi(\zeta) f(\zeta) d \mu(\zeta)=\int h(\xi) \int f(\zeta) d \mu_{\xi}(\zeta) d v(\xi)
$$

If $R$ is smooth, each $\mu_{\xi}$ may be chosen concentrated in $\pi^{-1}(\xi)$.

Proof. If $Z$ is countably or uncountably infinite, it is Borel isomorphic to the one-point compactification of the integers, or to the Cantor set, respectively. Thus we may let $Z$ have a zero-dimensional, compact, metrizable topology. Let $\Sigma$ be the algebra of sets generated by a countable basis of compact open sets. $\Sigma$ is countable, and any decomposition of a set in $\Sigma$ into non empty disjoint sets in $\Sigma$ must be finite. It follows that any finitely additive, non-negative function on $\Sigma$ is a measure, and as $\Sigma$ generates the Borel structure on $Z$, extends uniquely to a measure on $Z$ (see [10, p. 54]).

For each Borel set $S$ in $\Sigma, C \rightarrow \mu\left(S \cap \pi^{-1}(C)\right)$ is absolutely continuous with respect to $v$, hence there is a non-negative Borel function $g_{S}$ on $Z / R$ with

$$
\mu\left(S \cap \pi^{-1}(C)\right)=\int_{C} g_{S}(\xi) d v(\xi)
$$

If $S$ and $S^{\prime}$ are disjoint sets in $\Sigma$,

$$
\mu\left(\left(S \cup S^{\prime}\right) \cap \pi^{-1}(C)\right)=\mu\left(S \cap \pi^{-1}(C)\right)+\mu\left(S^{\prime} \cap \pi^{-1}(C)\right),
$$

hence

$$
\int_{C} g_{S \cup S}(\xi) d v(\xi)=\int_{C}\left[g_{S}(\xi)+g_{S},(\xi)\right] d v(\xi)
$$

for all Borel $C$, and

$$
g_{s \cup s^{\prime}}(\xi)=g_{s}(\xi)+g_{s^{\prime}}(\xi)
$$

for all $v$-almost all $\xi$. If $S=Z$ in (7),

$$
v(C)=\int_{C} g_{\mathrm{z}}(\xi) d v(\xi)
$$

hence $g_{\mathrm{Z}}(\xi)=1 v$-almost everywhere. Letting the $g_{s}$ be zero on a Borel $v$-null set, we may assume $S \rightarrow g_{S}(\xi)$ is finitely additive, and extends to a measure $\mu_{\xi} \in M(Z)_{1}$.

The family of Borel sets $T$ in $Z$ for which $\xi \rightarrow \mu_{\xi}(T)$ is Borel and 


$$
\mu\left(T \cap \pi^{-1}(C)\right)=\int_{C} \mu_{\xi}(T) d v(\xi)
$$

for Borel $C$ in $Z / R$, is monotonic and contains $\Sigma$. Thus from [10, p. 27], the family consists of all Borel sets in $Z$. If $f$ and $h$ are Borel characteristic functions, $\xi \rightarrow \int f d \mu_{\xi}$ is Borel, and (6) is true. Linearity implies the same for simple $f$ and $h$, i.e., finite linear combinations of Borel characteristic functions. If $f$ and $h$ are as described in the lemma, choose simple $f_{n}$ and $h_{n}$ with $f_{n} \rightarrow f, h_{n} \rightarrow h$ pointwise, and $\left|f_{n}\right| \leqq|f|,\left|h_{n}\right| \leqq|h|$. As $f$ is bounded, it is $\mu_{\xi}$-integrable, and $\int f_{n} d \mu_{\xi} \rightarrow \int f d \mu_{\xi}$. Thus $\xi \rightarrow \int f d \mu_{\xi}$ is Borel, and as $f$ was arbitrary, $\xi \rightarrow \mu_{\xi}$ is Borel. As $\xi \rightarrow \int|f| d \mu_{\xi}$ is bounded, $|h(\xi)| \int|f| d \mu_{\xi}$ is a $v$-integrable function dominating the $h_{n}(\xi) \int f_{n} d \mu_{\xi}$. Similarly, $|h \circ \pi(\zeta) f(\zeta)|$ is $\mu$-integrable and dominates $h_{n} \circ \pi(\zeta) f(\zeta)$. Taking limits, we obtain (6).

If $D$ is Borel in $Z / R$, we have from (8)

$$
\int_{C} \chi_{D}(\xi) d v(\xi)=v(D \cap C)=\int_{C} \mu_{\xi}\left(\pi^{-1}(D)\right) d v(\xi) .
$$

Thus

$$
\mu_{\xi}\left(\pi^{-1}(D)\right)=\chi_{D}(\xi)
$$

$v$-almost everywhere. If $\Delta$ is a countable separating algebra of sets in $Z / R$, let $N$ be Borel with $v(N)=0$ and (9) valid for $D \in \Delta$ and $\xi \notin N$. If $\xi \notin N$, choose $D_{1} \supseteq D_{2} \supseteq \cdots$ in $\Delta$ with $\bigcap D_{i}=\{\xi\}$. Then $\mu_{\xi}\left(\pi^{-1}\left(D_{i}\right)\right)=1$ implies that $\mu_{\xi}\left(\pi^{-1}(\xi)\right)=1$, i.e., $\mu_{\xi}$ is concentrated in $\pi^{-1}(\xi)$. Letting $\mu_{\xi}=0$ for $\xi \in N$, we are done.

LeMma 4.5 ([16, THEOREM 2.11]). Say that $Z$ is standard Borel space with a Borel field $\mathfrak{X}: Z \rightarrow \mathscr{A}_{u}$. If $R$ is an equivalence relation on $Z$, and $\mu$ is a measure on $Z$, let $\mu=\int \mu_{\xi} d v(\xi)$ be a decomposition of the type described in Lemma 4.4. Then there is a spatial isomorphism

$$
\int \mathfrak{U}(\zeta) d \mu(\zeta) \cong \int^{\beta} \mathfrak{A}\left(\mu_{\xi}\right) d v(\xi)
$$

where $\beta(\xi)=\gamma\left(\mu_{\xi}\right), \gamma$ a coherence for $\mu \rightarrow \mathfrak{S}(\mu)$ as described in Lemma 4.3.

Proof. Let $\mathfrak{H}: Z \rightarrow \mathscr{H}$ be the underlying field of Hilbert spaces for $\mathfrak{A}$. $\mathfrak{S}$ is Borel as $Z_{n}=\left\{\zeta: \mathfrak{U}(\zeta) \in \mathscr{A}_{n}\right\}$ is Borel. We must define an isometry

$$
U: \mathfrak{T}(\mu) \rightarrow \int \mathfrak{H}^{\beta}\left(\mu_{\xi}\right) d v(\xi)
$$

Let $\mathfrak{B}$ be the uniformly bounded Borel fields $x: Z \rightarrow \mathfrak{H}_{u}$ in $\mathfrak{H}$, and for each $\lambda \in M(Z)$, let $\mathfrak{B}(\lambda)$ be the corresponding subspace of $\mathfrak{H}(\lambda)$. From Lemma 4.3, if $x \in \mathfrak{B}$, $\lambda \rightarrow x(\lambda)$ is a uniformly bounded $\gamma$-Borel field in $\mathfrak{S}(\lambda)$ on $M(Z)_{1}$. Thus $\xi \rightarrow x\left(\mu_{\xi}\right)$ is uniformly bounded and $\beta$-Borel in $\mathfrak{S}\left(\mu_{\xi}\right)$, and 
We have

$$
x\left(\mu_{\xi}\right)^{\beta}(v) \in \int \mathfrak{H}^{\beta}\left(\mu_{\xi}\right) d v(\xi)
$$

$$
\begin{aligned}
\left\|x\left(\mu_{\xi}\right)^{\beta}(v)\right\|^{2} & =\int\left\|\beta(\xi) x\left(\mu_{\xi}\right)\right\|^{2} d v(\xi) \\
& =\int\left\|x\left(\mu_{\xi}\right)\right\|^{2} d v(\xi) \\
& =\iint\|x(\zeta)\|^{2} d \mu_{\xi}(\zeta) d v(\xi) \\
& =\int\|x(\zeta)\|^{2} d \mu(\zeta) \\
& =\|x(\mu)\|^{2} .
\end{aligned}
$$

Defining $U$ on $\mathfrak{B}(\mu)$ by $U(x(\mu))=x\left(\mu_{\xi}\right)^{\beta}(v), U$ is an isometry.

As in the proof of Lemma 4.3, we may select uniformly bounded Borel functions $g_{i}$ on $Z$ which span $L^{\infty}(\lambda)$ for all $\lambda \in M(Z)$. The functions $h_{i}(\xi)=\int g_{i}(\zeta) d \mu_{\xi}(\zeta)$ are bounded and Borel on $Z / R$. They span $L^{\infty}(v)$, as if $h \in L^{1}(v)$ and $h \perp h_{i}$ for all $i$,

$$
0=\int h h_{i} d v=\int h \circ \pi(\zeta) \bar{g}_{i}(\zeta) d \mu(\zeta)
$$

i.e., $h \circ \pi=0 \mu$-almost everywhere, and $h=0 v$-almost everywhere. If $h$ is a uniformly bounded Borel function on $Z / R$, we assert that the class $h \circ \pi\left(\mu_{\xi}\right)$ is that of the constant $h(\xi)$ for $v$-almost all $\xi$. If $C$ is Borel in $Z / R$, letting the $h$ of (6) be $\chi_{c}$, and then $\chi_{c} h$,

$$
\int_{C} \int h \circ \pi(\zeta) \bar{g}_{i}(\zeta) d \mu_{\xi}(\zeta) d v(\xi)=\int_{C} h(\xi) \int \bar{g}_{i}(\zeta) d \mu_{\xi}(\zeta) d v(\xi),
$$

hence there is a Borel set $N$ in $Z / R$ with $v(N)=0$ and

$$
\int h \circ \pi(\zeta) \bar{g}_{i}(\zeta) d \mu_{\xi}(\zeta)=\int h(\xi) \bar{g}_{i}(\zeta) d \mu_{\xi}(\zeta)
$$

for all $\xi \notin N$. As the $g_{i}$ span $L^{\infty}\left(\mu_{\xi}\right), h \circ \pi(\zeta)=h(\xi)$ for $\mu_{\xi}$-almost all $\zeta, \xi \notin N$.

Again from the proof of Lemma 4.3, we may choose uniformly bounded Borel vector fields $v_{j}$ in $\mathfrak{S}$ with $v_{j}(\lambda)$ spanning $\mathfrak{S}(\lambda)$ for all $\lambda \in M(Z)$. From Lemma 4.2, the vectors

$$
h_{i}(v) v_{j}\left(\mu_{\xi}\right)^{\beta}(v)=U\left(h_{i} \circ \pi(\mu) v_{j}(\mu)\right)
$$

span $\int \mathfrak{H}^{\beta}\left(\mu_{\xi}\right) d v(\xi)$. As the $v_{j}(\mu)$ span $\mathfrak{S}(\mu), \mathfrak{B}(\mu)$ and its image are dense, and $U$ extends to the desired isometry. 
If $A$ is a uniformly bounded Borel field in $\mathfrak{A}$, we have from Lemma 4.2 that $\xi \rightarrow A\left(\mu_{\xi}\right)$ is a uniformly bounded $\beta$-Borel field in $\mathfrak{U}\left(\mu_{\xi}\right)$, and

$$
A\left(\mu_{\xi}\right)^{\beta}(v) \in \int^{\beta} \mathfrak{A}\left(\mu_{\xi}\right) d v(\xi) .
$$

If $x$ is in $\mathfrak{B},(\bar{A} x)(\zeta)=A(\zeta) x(\zeta)$ is another field in $\mathfrak{B}$,

$$
\begin{aligned}
U A(\mu) x(\mu) & =U(\bar{A} x)(\mu) \\
& =\left[(\bar{A} x)\left(\mu_{\xi}\right)\right]^{\beta}(v) \\
& =\left[\beta(\xi) A\left(\mu_{\xi}\right) x\left(\mu_{\xi}\right)\right](v) \\
& =\left[A\left(\mu_{\xi}\right)^{\beta} x\left(\mu_{\xi}\right)^{\beta}\right](v) \\
& =\left[A\left(\mu_{\xi}\right)^{\beta}(v)\right] x\left(\mu_{\xi}\right)^{\beta}(v),
\end{aligned}
$$

hence $U A(\mu) U^{-1}=A\left(\mu_{\xi}\right)^{\beta}(v)$. As

$$
U\left(h_{i} \circ \pi(\mu) x(\mu)\right)=h_{i}(v) x\left(\mu_{\xi}\right)^{\beta}(v),
$$

$U h_{i} \circ \pi(\mu) I U^{-1}=h_{i}(v) I$.

Letting $B_{k}(\zeta)$ be an enumeration of the fields $f_{l}(\zeta) I$ and $A_{j}(\zeta)$ described in the proof of Lemma 4.3, the $B_{k}(\lambda)$ generate $\mathfrak{A}(\lambda)$ for each $\lambda \in M(Z)$. From Lemma 4.2, the $B_{k}\left(\mu_{\xi}\right)^{\beta}(v)$, together with the operators $h_{i}(v) I$, generate $\int^{\beta} \mathfrak{A}\left(\mu_{\xi}\right) d v(\xi)$. As these are the images of the $B_{k}(\mu)$ and the $h_{i} \circ \pi(\mu) I$, respectively, $U$ defines a spatial isomorphism of $\int \mathfrak{U}(\zeta) d \mu(\zeta)$ onto $\int^{\beta} \mathfrak{A}\left(\mu_{\xi}\right) d v(\xi)$.

5. Central decompositions. If $\mathfrak{A}$ is a von Neumann algebra on a separable Hilbert space, a central decomposition $(Z, \mathscr{S}, \mu, \mathfrak{F})$ of $\mathfrak{A}$ is a standard Borel space $(Z, \mathscr{S})$ with a measure $\mu$ and a Borel field $\mathfrak{F}: Z \rightarrow \mathscr{F}$ with

$$
\mathfrak{U} \cong \int \mathfrak{F}(\zeta) d \mu(\zeta)
$$

Such a decomposition always exists, and if $\left(Z_{0}, \mathscr{S}_{0}, \mu_{0}, \mathfrak{F}_{0}\right)$ is another such decomposition, there is a measure isomorphism $\left(T, N, N_{0}\right)$ of $Z$ and $Z_{0}$ (see $\S 4$ ) with $\mathfrak{F}(\zeta) \cong \mathfrak{F}_{0}(T(\zeta))$ for all $\zeta \in Z-N$. Letting $\pi_{1}$ be the quotient map of $\mathscr{F}$ onto $\hat{\mathscr{F}}, \pi_{1} \circ \mathfrak{F}$ and $\pi_{1} \circ \mathfrak{F}_{0}$ map $\mu$ and $\mu_{0}$, respectively, onto null-set equivalent measures $\hat{\mu}$ and $\hat{\mu}_{0}$ on $\hat{\mathscr{F}}$. We say that $\mathfrak{A}$ is centrally smooth if there is a Borel set $P \subseteq \hat{\mathscr{F}}$ with $\mu(P)=0$ and $\hat{\mathscr{F}}-P$ countably separated. Enlarging $P$ by a Borel null-set, we may assume $\hat{\mathscr{F}}-P$ is standard (see $[18$, Theorem 6.1$]$ ), hence we call such measures standard.

If $Z$ is a Borel space, let $M_{0}(Z)$ be the continuous measures on $Z$, i.e., those measures for which all one-point sets have zero mass. For cardinals $m$ with $1 \leqq m \leqq \infty=\aleph_{0}$, let $M_{m}(Z)$ be the measures totally concentrated in $m$ points. Given an arbitrary measure $\mu$, there exist unique measures $\mu^{c}$ and $\mu^{d}$, with 
$\mu=\mu^{c}+\mu^{d}$, where $\mu^{c}$ is continuous, and $\mu^{d}$ is discrete, i.e., totally concentrated in points.

If $\mu \in M(Z)$, define

$$
\|\mu\|=\sup \{|\mu(\{\zeta\})|: \zeta \in Z\} .
$$

LEMMA 5.1. If $Z$ is countably separated, then $\mu \rightarrow\|\mu\|$ is Borel on $M(Z)$.

Proof. Choose a sequence of Borel sets $S_{k}$ separating $Z$. Let $S_{k}^{0}=S_{k}$, $S_{k}^{1}=Z-S_{k}, S(\varnothing)=Z$, and for any sequence $i_{1}, \cdots, i_{n}$ of zeros and ones, let

$$
S\left(i_{1}, \cdots, i_{n}\right)=S_{1}^{i_{1}} \cap \cdots \cap S_{n}^{i_{n}}
$$

If $\mu \in M(Z)$, define

$$
\|\mu\|_{n}=\max \mu\left(S\left(i_{1}, \cdots, i_{n}\right)\right), \quad i_{1}, \cdots, i_{n}=0,1 .
$$

$\|\mu\|_{n}$ is a decreasing sequence, hence its limit exists. As $\mu \rightarrow\|\mu\|_{n}$ is Borel, it will suffice to prove that for all $\mu \in M(Z),\|\mu\|=\lim \|\mu\|_{n}$.

If $S$ is Borel, define the measure $\mu \mid S$ by $(\mu \mid S)(T)=\mu(S \cap T)$. If $\lim \|\mu\|_{n}=\delta>0$, define a sequence $j_{0}, j_{1}, \cdots$ as follows. Let $j_{0}=\varnothing$. Suppose that $j_{0}, \cdots, j_{r}$ have been defined with

$$
\lim \left\|\mu \mid S\left(j_{1}, \cdots, j_{r}\right)\right\|_{n}=\delta .
$$

As the sequences

$$
\begin{aligned}
& a_{n}=\left\|\mu \mid S\left(j_{1}, \cdots, j_{r}, 0\right)\right\|_{n-1}, \\
& b_{n}=\left\|\mu \mid S\left(j_{1}, \cdots, j_{r}, 1\right)\right\|_{n-1}
\end{aligned}
$$

are decreasing, and

$$
\left\|\mu \mid S\left(j_{1}, \cdots, j_{r}\right)\right\|_{n}=\max \left\{a_{n}, b_{n}\right\}
$$

$\lim \left\|\mu \mid S\left(j_{1}, \cdots, j_{r}\right)\right\|_{n}$ must coincide with either $\lim a_{n}$ or $\lim b_{n}$. This provides us with $j_{r+1}$ satisfying (10). As the sets $S_{k}$ separate $Z, \bigcap_{r} S\left(j_{1}, \cdots, j_{r}\right)$ has at most one point. On the other hand,

$$
\mu\left(S\left(j_{1}, \cdots, j_{r}\right)\right) \geqq\left\|\mu \mid S\left(j_{1}, \cdots, j_{r}\right)\right\|_{n} \geqq \delta ;
$$

hence the intersection must contain a point $\zeta$ with $\mu(\{\zeta\}) \geqq \delta$. We conclude that $\|\mu\| \geqq \lim \|\mu\|_{n}$. The converse inequality is trivial.

LEMMA 5.2. If $Z$ is countably separated, the sets $M_{m}(Z)$ are Borel in $M(Z)$, and the maps $\mu \rightarrow \mu^{c}$ and $\mu \rightarrow \mu^{d}$ are Borel.

Proof. If $\delta>0$ and $\mu \in M(Z)$, there are at most finitely many $\zeta$ with $\mu(\{\zeta\}) \geqq \delta$. Thus there always exist $\zeta \in Z$ with $\mu(\{\zeta\})=\|\mu\|$. We call such $\zeta$ maximal $\mu$-atoms. Define $S\left(i_{1}, \cdots, i_{n}\right)$ as in the proof of Lemma 5.1. For each $\mu \in M(Z)$, define 
$i_{0}(\mu)=\varnothing$. Suppose that $i_{0}(\mu), \cdots, i_{n}(\mu)$ have been defined with $S\left(i_{1}(\mu), \cdots, i_{n}(\mu)\right)$ containing a maximal $\mu$-atom. Let $i_{n+1}(\mu)=0$ or 1 be the least integer with $S\left(i_{1}(\mu), \cdots, i_{n+1}(\mu)\right)$ containing a maximal $\mu$-atom. Define

$$
\mu^{a}=\lim \mu \mid S\left(i_{1}(\mu), \cdots, i_{n}(\mu)\right),
$$

where limit is taken in the sense of convergence of the corresponding integrals of each bounded Borel function. $\mu^{a}$ is the "part" of $\mu$ concentrated in some maximal $\mu$-atom. It will suffice to show that $\mu \rightarrow \mu^{a}$ is Borel. For then, letting $c_{1}(\mu)=\mu-\mu^{a}$, and $c_{n+1}(\mu)=c_{1}\left(c_{n}(\mu)\right)$,

$$
\begin{aligned}
M_{0}(Z) & =\left\{\mu: c_{1}(\mu)=\mu\right\}, \\
\bigcup_{1 \leqq k \leqq n} M_{k}(Z) & =\left\{\mu: c_{n}(\mu)=0\right\} \quad(n<\infty), \\
\bigcup_{1 \leqq k \leqq \infty} M_{k}(Z) & =\left\{\mu: \lim c_{n}(\mu)=0\right\}, \\
\mu^{c} & =\lim c_{n}(\mu) .
\end{aligned}
$$

If $n=0, \mu \rightarrow \mu \mid S\left(i_{1}(\mu), \cdots, i_{n}(\mu)\right)=\mu$ is Borel. Suppose that we have proved $\mu \rightarrow \mu \mid S\left(i_{1}(\mu), \cdots, i_{n}(\mu)\right)$ is Borel. Then the maps

$$
\mu \rightarrow \mu\left|S\left(i_{1}(\mu), \cdots, i_{n}(\mu), j\right)=\mu\right| S\left(i_{1}(\mu), \cdots, i_{n}(\mu)\right) \cap S_{n}^{j}, \quad j=0,1,
$$

are Borel. From Lemma 5.1,

$$
g(\mu)=\|\mu\|-\left\|\mu \mid S\left(i_{1}(\mu), \cdots, i_{n}(\mu), 0\right)\right\|
$$

is Borel. Letting $h(0)=1$ and $h(t)=0$ for real $t \neq 0$,

$$
\begin{aligned}
& \mu \rightarrow \mu \mid S\left(i_{1}(\mu), \cdots, i_{n}(\mu), i_{n+1}(\mu)\right) \\
& \quad=h(g(\mu)) \mu\left|S\left(i_{1}(\mu), \cdots, i_{n}(\mu), 0\right)+[1-h(g(\mu))] \mu\right| S\left(i_{1}(\mu), \cdots, i_{n}(\mu), 1\right)
\end{aligned}
$$

is Borel. From (11) it follows that $\mu \rightarrow \mu^{a}$ is Borel.

Let $\mathfrak{P}_{0}=L^{\infty}([0,1], v)$ where $v$ is Lebesque measure. If $1 \leqq m \leqq \infty=\aleph_{0}$, let $\mathfrak{P}_{m}=L\left(J_{m}^{\infty}, v_{m}\right)$, where $J_{m}$ are the first $m$ integers, and $v_{m}$ is the measure with $v_{m}(i)=2^{-i}$. The $\mathfrak{P}_{m}$ are von Neumann algebras when they are represented on the corresponding $L^{2}$-spaces. If $\mu$ is a standard continuous or discrete measure acting on a Borel space $Z, L^{\infty}(Z, \mu)$ represented on $L^{2}(Z, \mu)$ is spatially isomorphic to one of these algebras.

THEOREM 5.3. If $\mathfrak{U}$ is a centrally smooth von Neumann algebra, there exists a Borel subset $W$ of $\hat{\mathscr{F}}$, a Borel map $\mathfrak{B}: W \rightarrow \mathscr{F}$ with $\pi_{1} \circ \mathfrak{B}(\xi)=\xi$, and standard measures $v_{m}$ on $W$ for which

$$
\mathfrak{U} \cong \sum_{m=0}^{\infty} \int \mathfrak{B}(\xi) d v_{m}(\xi) \otimes \mathfrak{P}_{m} .
$$

The measures $v_{m}$ with $m>0$ may be chosen disjoint. 
Proof. Let $(Z, \mathscr{S}, \mu, \mathfrak{F})$ be a central decomposition for $\mathfrak{A}$, and $\hat{\mu}$ be the induced measure on $\hat{\mathscr{F}}$. As $\hat{\mu}$ is standard, we may choose a Borel set $W \subseteq \hat{\mathscr{F}}$ with $\hat{\mu}(\hat{\mathscr{F}}-W)=0$, and $W$ standard in the relative structure. Decreasing $W$ by a Borel null set, we may let $\mathfrak{B}: W \rightarrow \mathscr{F}$ be a Borel cross-section for $\pi_{1}$ (see $[18$, Theorem 6.3]). Letting $Z_{0}$ be the $\pi_{1} \circ \mathfrak{F}$ inverse image of $W$, the $Z_{0}$ quotient structure must correspond to the relative structure on $W$, as the latter is countably separated (see the proof of [18, Theorem 5.1]). From Lemmas 4.4 and 4.5, we have a Borel map $\xi \rightarrow \mu_{\xi}$ of $W$ into $M\left(Z_{0}\right)_{1}$ with $\mu_{\xi}$ concentrated in $\pi_{1}^{-1}(\xi)$ and

$$
\mathfrak{A} \cong \int^{\beta} \mathfrak{F}\left(\mu_{\xi}\right) d \hat{\mu}(\xi) .
$$

As $\mathfrak{F}\left(\mu_{\xi}\right) \cong \mathfrak{F}\left(\mu_{\xi}^{c}\right) \oplus \mathfrak{F}\left(\mu_{\xi}^{d}\right)$, we have from Lemma 4.1 and (1)

$$
\mathfrak{A} \cong \int^{\beta^{c}} \mathfrak{F}\left(\mu_{\xi}^{c}\right) d \mu(\xi) \oplus \int^{\beta^{d}} \mathfrak{F}\left(\mu_{\xi}^{d}\right) d \mu(\xi),
$$

where $\beta^{c}(\xi)=\gamma\left(\mu_{\xi}^{c}\right)$ and $\beta^{d}(\xi)=\gamma\left(\mu_{\xi}^{d}\right)$. Let $v_{0}$ be the restriction of $\hat{\mu}$ to $W_{0}=\left\{\xi: \mu_{\xi}^{c} \neq 0\right\}$ and for $m \geqq 1$, let $v_{m}$ be the restriction of $\hat{\mu}$ to $\left\{\xi: \mu_{\xi}^{d} \in M_{m}\left(Z_{0}\right)\right\}$. Then from Lemma 4.1 and (3),

$$
\mathfrak{F}\left(\mu_{\xi}^{c}\right) \cong \mathfrak{B}(\xi) \otimes \mathfrak{P}_{0}
$$

for $v_{0}$-almost all $\xi$, and for $m \geqq 1$,

$$
\mathfrak{F}\left(\mu_{\xi}^{d}\right) \cong \mathfrak{B}(\xi) \otimes \mathfrak{P}_{m}
$$

for $v_{m}$-almost all $\xi$. The theorem follows from (12) and (2).

It is readily verified that the measure classes of the $v_{m}$ are uniquely determined by $\mathfrak{A}$, and that any such system of standard measures arises in this manner from a centrally smooth von Neumann algebra. The latter is in contrast with the group representation situation, in which "noncanonical" standard measures can occur (see $[18$, p. 164]).

6. Global structure. Let $\mathfrak{A}$ be a von Neumann algebra on a Hilbert space $\mathfrak{H}$, 3 the Boolean algebra of central projections in $\mathfrak{U}$. We say $E, F \in \mathcal{Z}$ are spatially equivalent, $E \cong F$, if $\mathfrak{U} E$ and $\mathfrak{U} F$ are spatially isomorphic (a corresponding theory may be formulated using algebraic isomorphisms). Letting $M(\mathfrak{U})$ be the operators $T$ on $\mathfrak{S}$ with $T \mathfrak{A} T^{*} \subseteq \mathfrak{U}$ and $T^{*} \mathfrak{U} T \subseteq \mathfrak{U}$, we have $E \cong F$ if and only if there is a partial isometry $U \in M(\mathfrak{A})$ with $U^{*} U=E$ and $U U^{*}=F .3$, together with the equivalence $\cong$, may be thought of as a global analogue of the lattice of all projections in $\mathfrak{U}$ with the usual equivalence. More precisely, $(3, \cong)$ is a dimension lattice in the sense of Loomis [14], and we may examine all of the concepts normally associated with such a lattice.

Projections $E, F \in 3$ are globally disjoint if there do not exist $E_{1}, F_{1} \in \mathcal{Z}$ with $0 \neq E_{1} \leqq E, 0 \neq F_{1} \leqq F$ and $E_{1} \cong F_{1} . E$ is globally central if $E$ and $I-E$ 
are globally disjoint. We let $\mathcal{Z}_{G}$ be the collection of such projections. If $E \in \mathcal{Z}_{\sigma}$ and $F \cong E, F \leqq E$.

LEMMA 6.1. $3_{G}$ consists of the central projections left fixed by the spatial automorphisms of $\mathfrak{A}$.

Proof. If $E$ is globally central, and $V$ induces a spatial automorphism of $\mathfrak{A}$, then $V^{*} E V \cong E$ and $V E V^{*} \cong E$ imply $V^{*} E V=E$. If $E$ is not globally central choose a nonzero partial isometry $U \in M(\mathfrak{A})$ with $U^{*} U \leqq E, U U^{*} \leqq I-E$. Then the unitary $V=U+U^{*}+\left[I-\left(U^{*} U+U U^{*}\right)\right]$ induces a spatial automorphism of $\mathfrak{A}$ that does not leave $E$ fixed.

If $E \in \mathcal{Z}$, we let $G(E)$ be the minimal projection in $\mathcal{Z}_{G}$ with $E \leqq G(E)$. We say $\mathfrak{A}$ is a global factor if $\mathfrak{Z}_{G}=\{0, I\}$, and $\mathfrak{A}$ is globally multiplicity free if $\mathfrak{Z}_{G}=\mathfrak{Z}$. $\mathfrak{U}$ is of global type $I$ if there is a projection $E \in 3$ with $G(E)=I$ and $E$ globally multiplicity free. $\mathfrak{A}$ is globally finite if $E \in \mathfrak{Z}$ and $E \cong I$ imply $E=I$. $\mathfrak{A}$ is globally semi-finite if there is a projection $E \in \mathfrak{Z}$ with $G(E)=I$ and $\mathfrak{A} E$ finite. $\mathfrak{A}$ is of global type II if $\mathfrak{A}$ is globally semi-finite, and there does not exist $E \in \mathcal{3}$ with $E \neq 0$ and $\mathfrak{A} E$ of global type I. Finally, $\mathfrak{A}$ is of global type III if there does not exist $E \in \mathfrak{Z}$ with $E \neq 0$ and $\mathfrak{A} E$ globally semi-finite.

It is a simple exercise to check that the centrally smooth global factors are those of the form $\mathfrak{F} \otimes \mathfrak{P}_{m}, \mathfrak{F}$ a factor, and that such an algebra has global type I if $m \geqq 1$, and global type III if $m=0$. Thus a global factor (or in fact, any von Neumann algebra) of global type II would not be centrally smooth. The existence of such an algebra would imply that $\hat{\mathscr{F}}$ and $\tilde{\mathscr{F}}$ are nonsmooth, and the existence of uncountably many nonalgebraically isomorphic factors would follow. We are currently examining the von Neumann algebras associated with the regular representations of countable discrete groups. We remark that it is not difficult to prove that if such a group has all of its automorphism classes (other than that of the identity) infinite, then the corresponding algebra is a global factor. The rationals under addition form such a group.

We have been unable to give a global characterization for the centrally smooth von Neumann algebras. In particular, we do not know if the analogue of Guichardet's theorem for multiplicity free representations [8] is true. Specifically, is any globally multiplicity free von Neumann algebra on a separable Hilbert space centrally smooth?

\section{BIBLIOGRAPHY}

1. J. Dieudonné, Sur le théorème de Lebesque-Nikodym. III, Ann. Univ. Grenoble (N.S.) 23 (1948), 25-53. 1957.

2. J. Dixmier, Les algebres d'opérateurs dans l'espace hilbertien, Gauthier-Villars, Paris,

3. - Dual et quasi-dual d'une algebre de Banach involutive, Trans. Amer. Math. Soc. 104 (1962), 278-283. 
4. E. Effros, Transformation groups and $C^{*}$-algebras, Ann. of Math. 81 (1965), 38-55.

5. - The Borel space of von Neumann algebras on a separable Hilbert space, Pacific J. Math. (to appear).

6. J. Feldman, Borel sets of states and of representations, Michigan Math. J. 12 (1965), 363-366.

7. R. Godement, Sur la théorie des representations unitaires, Ann. of Math. 53 (1951), 68-124.

8. A. Guichardet, Sur un problème posé par G. W. Mackey, C.R. Acad. Sci. Paris 250 (1960), 962-963.

9. P. R. Halmos, The decomposition of measures, Duke Math. J. 8 (1941), 386-392.

10. - Measure theory, Van Nostrand, New York, 1950.

11. - Introduction to Hilbert space and the theory of multiplicity, Chelsea, New York, 1951.

12. I. Kaplansky, A theorem on rings of operators, Pacific J. Math. 1 (1951), 227-232.

13. C. Kuratowski, Topologie. I, 3rd ed., Monagrafie Mat. 20, Warsaw, 1952.

14. L. H. Loomis, The lattice theoretic backgrcund of the dimension theory of operator algebras, Mem. Amer. Math. Soc. No. 18 (1955), 36 pp.

15. G. W. Mackey, Induced representations of locally compact groups. I, Ann. of Math. 55 (1952), 101-139.

16. ——, Induced representations of locally compact groups. II, Ann. of Math. 58 (1953), 193-221.

17. - The theory of group representations (notes by Fell and Lowdenslager), Lecture notes, Univ. of Chicago, Chicago, Ill., 1955.

18. —, Borel structures in groups and their duals, Trans. Amer. Math. Soc. 85 (1957), 134-165.

19. - Infinite-dimensional group representations, Bull. Amer. Math. Soc. 69 (1963), $628-686$.

20. J. von Neumann, Zur Operatorenmethode in der Klassischen Mechanik, Ann. of Math. 33 (1932), 789-791.

21. V. A. Rohlin, On the fundamental ideas of measure theory, Amer. Math. Soc. Transl. No. 71, 1952, $55 \mathrm{pp}$.

22. J. Schwartz, Two finite, non-hyperfinite, non-isomorphic factors, Comm. Pure Appl. Math. 16 (1963), 19-26.

23. - Non-isomorphism of a pair of factors of type III, Comm. Pure Appl. Math. 16 (1963), 111-120.

\section{University of Pennsylvania, Philadel phia, Pennsylvania}

